\title{
A GSC EMPLOYING TWO-DIMENSIONAL FREQUENCY INVARIANT FILTERS
}

\author{
Wei Liu, Stephan Weiss, and Lajos Hanzo \\ Communications Research Group \\ School of Electronics \& Computer Science \\ University of Southampton, U. K. \\ $\{$ w. liu,s.weiss, l.hanzo\}@ecs.soton.ac.uk
}

\begin{abstract}
In this paper, based on the generalised sidelobe canceller (GSC), we propose a novel broadband adaptive beamformer, where its quiescent vector and blocking matrix are replaced by a series of two dimensional frequency invariant filters (FIFs). The new quiescent vector forms a beam pointing to the signal of interest, whereas the new blocking matrix forms a number of beams pointing to other directions, with a zero response to the signal of interest. As opposed to standard beamspace techniques, the FIFs need not have a very low sidelobe level, and the number of FIFs that can be implemented also increases. Compared with the traditional GSC, a faster convergence speed is achieved in addition to a resultant frequency-invariant beam pattern, as shown by simulations.
\end{abstract}

\section{INTRODUCTION}

In broadband beamforming, for artays to accomplish nulling over wide percentage bandwidth, tapped-delay lines are employed, resulting in a beamformer with $M$ sensors and tappeddelay lines of length $J$ as shown in Fig. 1. We need to employ a large number of sensors and long tapped-delay lines to achieve high interference rejection and resolution, which unavoidably increases the computational complexity of the adaptive part and slows down the convergence of the system. To overcome this problem, several methods have been proposed, such as the use of adaptive pole-zero filters in [1] and subband adaptive methods [2, 3]. Another method is broadband beamspace adaptive arrays [4], where several frequency invariant beams are formed pointing to different directions by a fixed beamforming network with two-dimensional (2-D) filters; thereafter an adaptive filter is used to combine these beam outputs to form the desired output. Since both the number of beams and that of the selected beams are small, the total number of adaptive weights are greatly reduced compared to the tapped-delay line system.

In this paper, we apply the broadband beamspace technique to the generalised sidelobe canceller (GSC) $[5,6]$, and propose a novel broadband adaptive beamformer, where the quiescent vector and the column vectors of the blocking matrix are replaced by a series of two dimensional frequency invariant filters (FIFs). The new quiescent vector forms a beam pointing to the direction of the signal of interest, whereas the new blocking matrix forms a number of beams pointing to the other directions with a zero response to the signal of interest.

In this new arrangement, as long as the FIFs have a good frequency invariant property, it is not necessary for them to have a very low sidelobe level. This is an advantage over the beamspace method in [4], where a low sidelobe level is required, resulting in a large dimension of the FIFs. Moreover, in the beamspace method, the main beam direction of the FIFs should ideally coincide with nulls (zero responses) of all other beams, which limits the number of FIFs that can be implemented and sacrifices too many degrees of freedom of the system. As a result, although [4] presents a fine approach, the interference cancellation capability is reduced compared to the traditional GSC method with tapped-delay lines. In our method, the FIFs in the blocking matrix only need to have one zero at the direction of the signal of interest, so we can implement a large number of FIFs and most of the interference cancellation capability of the array is re- 
served.

The paper is organised as follows. In Sec. 2 we will give a brief review of the GSC structure. In Sec. 3, the frequency invariant beamforming technique based on fan filters is reviewed and then we propose the GSC with two dimensional FIFs. The method to design the blocking matrix FIFs is described in Sec. 4. Finally, simulation results are given in Sec. 5 and conclusions drawn in Sec. 6 .

\section{GENERALISED SIDELOBE CANCELLER}

A linearly constrained minimum variance (LCMV) beamformer [7] performs the minimisation of the variance of the output signal with respect to some given spatial and spectral constraints. For the beamformer shown in Fig. 1, the output $e[n]$ can be expressed as

$$
e[n]=\mathbf{w}^{H} \mathbf{x},
$$

where the vector $\mathbf{x}$ holds received signal samples at the tapped-delay lines and $\mathbf{w}$ holds the corresponding weights.

The LCMV problem can be formulated as

$$
\min _{\mathbf{w}} \mathbf{w}^{H} \mathbf{R}_{x: \mathbf{w}} \mathbf{w} \quad \text { subject to } \quad \mathbf{C}^{H} \mathbf{w}=\mathbf{f},
$$

where $\mathbf{R}_{x: x}$ is the covariance matrix of observed array data in $\mathbf{x}, \mathbf{C} \in \mathbb{C}^{M J \times J}$ is the constraint matrix and $\mathbf{f}$ is the response vector.

The constrained optimisation of the LCMV problem in (2) can be conveniently solved using a GSC, which performs a projection of the data onto an unconstrained subspace by means of a blocking matrix $\mathbf{B}$ and a quiescent

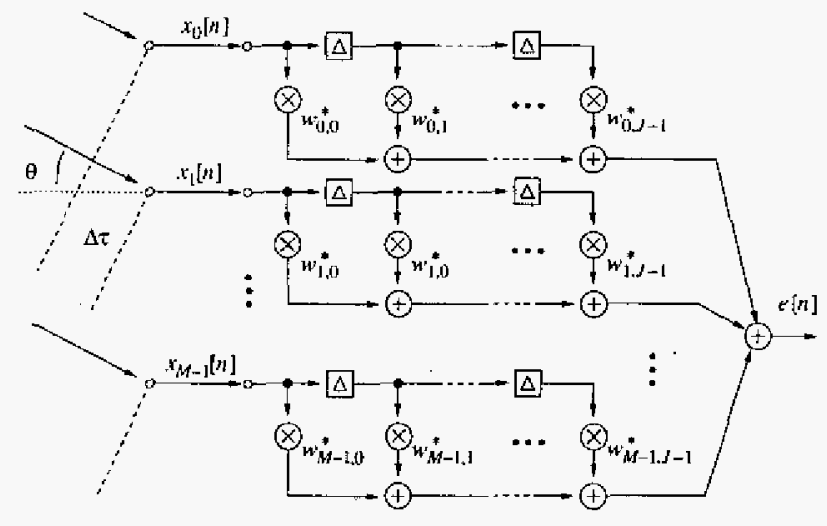

Fig. 1. A broadband beamformer with $M$ sensors, each followed by a $J$-tap filter.

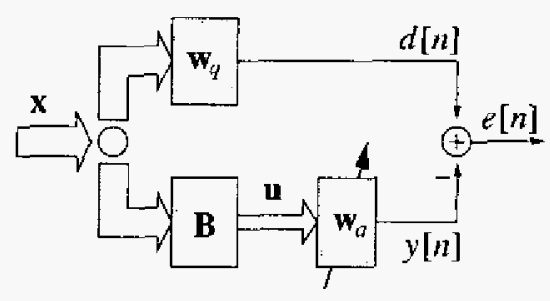

Fig. 2. A generalised sidelobe canceller structure.

vector $\mathbf{w}_{\mathrm{q}}$. Thereafter, standard unconstrained optimisation algorithms such as least mean square (LMS) or recursive least squares (RLS) algorithms can be invoked [8]. Fig. 2 shows its principle, where $\mathbf{u}=\mathbf{B}^{H} \mathbf{X}$ and

$$
d[n]=\mathbf{w}_{q}^{H} \mathbf{x} \quad \text { with } \quad \mathbf{w}_{q}=\mathbf{C}\left(\mathbf{C}^{H} \mathbf{C}\right)^{-1} \mathbf{f} .
$$

Since $\mathbf{w}_{q}$ is designed to satisfy the specified constraints, the signal of interest will pass through the beamformer with desired response independent of $\mathbf{w}_{\alpha}$. In the lower branch, the blocking matrix is required to block the signal of interest so that only interfering signals and noise exist. When adapting $\mathbf{w}_{a}$ to minimise the variance of the output signal $e[n]$, the scheme will tend to cancel the interference and noise component from $d[n]$.

Next, we will review the frequency invariant beamforming technique based on fan filters and then propose a new construction of the quiescent vector and the blocking matrix.

\section{GSC EMPLOYING TWO DIMENSIONAL FREQUENCY INVARIANT FILTERS}

Consider an impinging signal $e^{-j \omega t}$ with DOA angle $\theta$ as in Fig. 1, where the waveform arrives with a time delay $\Delta \tau$ at adjacent sensors separated by $d$ in a medium with propagation speed $c$. The received phase vector at the sensor array, $\underline{\mathbf{x}}$, is

$$
\underline{\mathbf{x}}=\left[1 e^{-j \omega \Delta \tau}, \cdots e^{-j \omega(M-1) \Delta T}\right]^{T} \text { with } \Delta \tau=\frac{d}{c} \sin \theta .
$$

Assume that the array sensors are spaced at half the wavelength of the maximum signal frequency and the temporal sampling frequency $\omega_{\mathrm{s}}$ is twice the maximum signal frequency. Then, we have $\Delta \tau=T_{s} \sin \theta$, where $T_{s}$ is the temporal sampling period. Noting $\omega T_{s}=\Omega$, where $\Omega$ is the 
normalised angular frequency, we obtain

$$
\mathbf{x}[n]=\left[\begin{array}{llll}
1 & e^{-j \Omega \sin \theta} & \cdots & e^{-j(M-1) \Omega \sin \theta}
\end{array}\right]^{T} \cdot e^{-j n \Omega} .
$$

The response of the beamformer to the discrete signal $e^{-j n \Omega}$ is

$$
R(\Omega, \theta)=\sum_{m=0}^{M-1} \sum_{k=0}^{J-1} w_{m, k}^{*} \cdot e^{-j m \Omega \sin \theta} \cdot e^{-j k \Omega} .
$$

With the substitution of $\Omega_{1}=\Omega$ and $\Omega_{2}=\Omega \sin \theta$, we obtain the two dimensional digital filter response

$$
R\left(\Omega_{1}, \Omega_{2}\right)=\sum_{m=0}^{M-1} \sum_{k=0}^{J-1} w_{m, k}^{*} \cdot e^{-j k \Omega_{1}} \cdot e^{-j m \Omega_{2}}
$$

As the spatio-temporal spectrum of the received signal lies on the line $\Omega_{2}=\Omega_{1} \sin \theta$, we can use the transformation $\tilde{\Omega}=\left(\frac{\Omega_{2}}{\Omega_{1}}-\sin \theta\right) \cdot \pi$ to get the coefficients $w_{m, k}^{*}$ from a one dimensional filter $H\left(e^{j \hat{\Omega}}\right)$. The resultant beamformer with such kind of coefficients will be frequency independent [4]. If $H\left(e^{j \bar{\Omega}}\right)$ is a lowpass filter [4], then signals from the directions around $\theta$ will correspond to its passband. In this case, a main beam is formed pointing to the direction $\theta$.

Applying this broadband beamforming technique to the GSC, we replace $\mathbf{w}_{q}$ by a two dimensional FIF with its main beam pointing to the signal of interest. For the blocking matrix $\mathbf{B}$, each column vector is replaced by a two dimensional FIF with a zero response to the signal of interest. By this arrangement, the broadband beamforming problem is transformed into a narrowband beamforming problem. If the filters are strictly frequency invariant, then we only need $M-1$ such filters in the blocking matrix and only one adaptive weight is required at each filter output. In practice, the FIFs are not ideally frequency invariant, therefore we may need to form more than $M-1$ such filters, or as an alternative, apply more than one adaptive weights at each of the outputs. However, the total number of adaptive weights should be much less than that of a traditional tapped-delay line system.

Without loss of generality, supposing the signal of interest comes from broadside, the main beam of $\mathbf{w}_{q}$ should point to the direction $\theta=0$. The blocking matrix is arranged such that its column vectors form $L$ FIFs with their main beam directions equally distributed over $\theta \in\left[-\frac{\pi}{2} ; 0\right)$ and the same number over $\theta \in\left(0 ; \frac{\pi}{2}\right]$. All these FIFs in the blocking matrix should have a zero response at $\theta=0$. Fig. 3 shows the arrangement of the $2 L+1$ main beams, which point to the directions $\theta_{l}=\frac{l \pi}{2 L}, l=-L, \cdots, 0, \cdots, L$, respectively. If the $2 L$ FIFs in the blocking matrix are strictly frequency invariant, $2 L$ should be equal to $M-1$. Here we set $L=\left\lceil\frac{M-1}{2}\right\rceil$, where $\lceil\cdot\rceil$ is the ceiling (or round-up) operator: When $M$ is odd, we have in total $M$ FIFs, while for even $M$, we obtain $M+1$ FIFs.

As mentioned, the FIF in the proposed method transforms the broadband beamforming problem into a narrowband beamforming problem. As long as the FIFs have a good frequency invariant property, it is not necessary for them to have a very low sidelobe level, which is different from the beamspace processing method [4], where a low sidelobe level is required, resulting in a large dimension of the FIFs and the requirement to increase the number of sensors. Moreover, in [4], the main direction of a beam should ideally coincide with nulls (zero responses) of all other beams. As a result, the interference cancellation capability is reduced comparing to the traditional GSC method. As an example, consider the case of a 5 -tap prototype FIR filter. Since this prototype has only 4 zeros, the maximum number of frequency invariant beams achieved in the beamspace method is 5, as shown in [4]. According to [4], to attain a good frequency invariant characteristic, the values of $M$ and $J$ need to be at least three times the length of the prototype filter, i.e. $M=J=15$ for the 5 tap prototype FIR filter. Comparing the number of beams, 5 , with the antenna number $M=15$, we may have sacrificed too many degrees of freedom of the system and a decreased performance is very likely to result in some circumstances. However, In our proposed method, the FIFs in the blocking matrix only need to have one zero in the direction of the signal of interest, so that a large number of FIFs can be implemented-in our case $2 L+1$ - even for a relatively small number of antenna elements $M$. Thus, by our approach, most of the interference cancellation capability of the array is retained. Compared to [4], the number of FIFs $N_{\text {FIF }}$ achieved by the beam space method and our proposed method is given by

$$
N_{\text {FIF }} \approx\left\{\begin{array}{ll}
\frac{M}{3} & \text { for beam space method } \\
2 \cdot\left\lceil\frac{M-1}{2}\right\rceil+1 & \text { for the proposed method }
\end{array},\right.
$$

where the selection of $\frac{M}{3}$ follows a design recommendation 


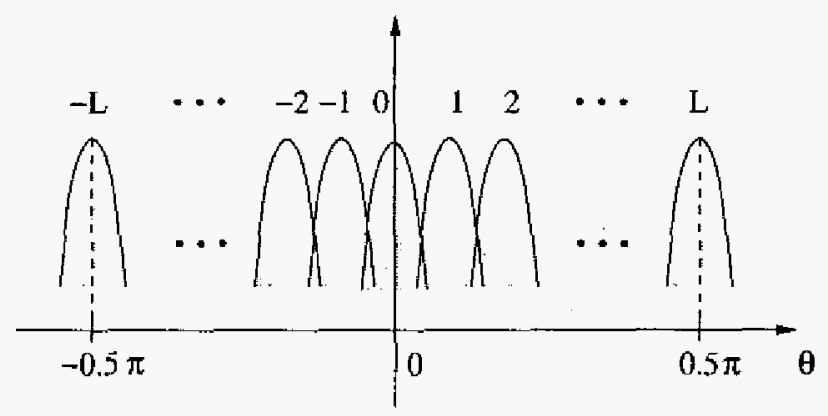

Fig. 3. An arrangement of the $2 L+1$ main beams.

in [4].

\section{DESIGN OF THE FREQUENCY INVARIANT FILTERS}

Since the FIF in $\mathbf{w}_{\varphi}$ is a normal 2-D FIF pointing to the direction $\theta_{s}$ of the signal of interest, it can be obtained by the fan filter method in [4]. We here focus on the design of the blocking matrix.

Suppose $h[k], k=0, \cdots, K-1$, is a linear-phase lowpass FIR filter with frequency response $H\left(e^{j \tilde{\Omega}}\right)$. The aim is to derive from it the two dimensional FIF with main beam in the direction $\theta_{i}$ and a zero response towards the direction $\theta_{s}$. Using the transformation $\tilde{\Omega}=\left(\frac{\Omega_{2}}{\Omega_{1}}-\sin \theta_{i}\right) \cdot \pi$, we obtain the frequency response of the FIF

$$
R\left(\Omega_{1}, \Omega_{2}\right)=H\left(e^{j\left(\frac{\Omega_{2}}{\Omega_{1}}-\sin \theta_{i}\right) \cdot \pi}\right) .
$$

As $\left.R\left(\Omega_{1}, \Omega_{2}\right)\right|_{\theta=\theta_{s}}=0$ and $\frac{\Omega_{2}}{\Omega_{1}}=\sin \theta_{s}$ in the direction $\theta=\theta_{s}$, we obtain

$$
\left.R\left(\Omega_{1}, \Omega_{2}\right)\right|_{\theta_{0}=\theta_{s}}=H\left(e^{j\left(\sin \theta_{s}-\sin \theta_{i}\right)-\pi}\right)=0 .
$$

Thus, the prototype filter design for the FIFs can be formulated as

$$
h[k]=\arg \min _{k[k]} \Phi_{l} \quad \text { subject to } \quad(10),
$$

where

$$
\Phi_{l}=\int_{\bar{\Omega}}^{\pi}\left(\left.H\left(e^{j \bar{\Omega}}\right)\right|^{2} d \bar{\Omega}\right.
$$

with $\bar{\Omega}_{y}$ being the stopband cutoff frequency. A constrained minimisation of $\Phi_{l}$ can be accomplished by invoking a nonlinear optimisation software package, such as the subroutines LCONF/DLCONF in the IMSL library [9].

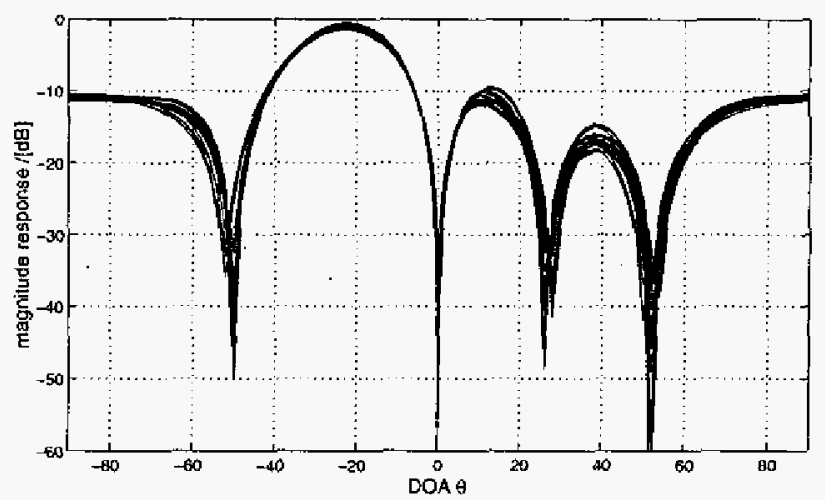

Fig. 4. Magnitude response of an FIF example over the band $\Omega \in[0.30 \pi ; 0.90 \pi]$.

Given this lowpass filter, we then follow the steps in [4]. The resultant FIF will have the desired zero response at $\theta_{s}$. The response of an $18 \times 18$ FIF over the band $\Omega \in$ $[0.30 \pi ; 0.90 \pi]$ derived from a 5 -tap lowpass filter with main beam pointing to $\theta=-22.5^{\circ}$ and zero response at $\theta=0$ is shown in Fig. 4.

\section{SIMULATION RESULTS}

In our simulation, the proposed GSC employing two dimensional FIFs with $M=J=18$ is compared with the traditional GSC with $M=18, J=15$. One adaptive weight is used for each of the FIF outputs. The signal of interest comes from broadside and with a signal to interference ratio (SIR) of $-20 \mathrm{~dB}$ and signal to noise ratio (SNR) of $20 \mathrm{~dB}$. Three interfering signals come from the angles of $20^{\circ},-40^{\circ}$ and $60^{\circ}$, respectively. Both the interfering signals and the signal of interest have a bandwidth of $[0.3 \pi ; 0.9 \pi]$. We use a normalised LMS algorithm for adaptation. The stepsizes are empirically chosen to achieve the same steadystate value of the mean square residual error (MSE). which are 0.23 for our FIF method and 0.38 for the traditional GSC. Simulation results are shown in Fig. 5, where the proposed method demonstrates a much faster convergence due to the transformation effect of the FIFs and the shorter adaptive filter length. Further, the proposed method automatically leads to a beam pattern with a good frequency invariant property, which is shown in Fig. 6 over the bandwidth $\Omega \in[0.3 \pi ; 0.9 \pi]$ and DOA range $\theta \in\left[-90^{\circ} ; 90^{\circ}\right]$, where the attenuation at the interferers' positions $20^{\circ},-40^{\circ}$ and 


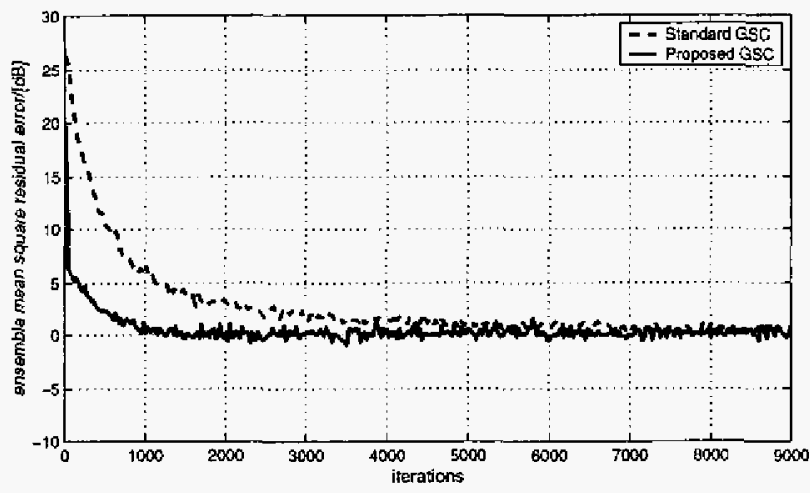

Fig. 5. Comparison of learning curves for our simulation.

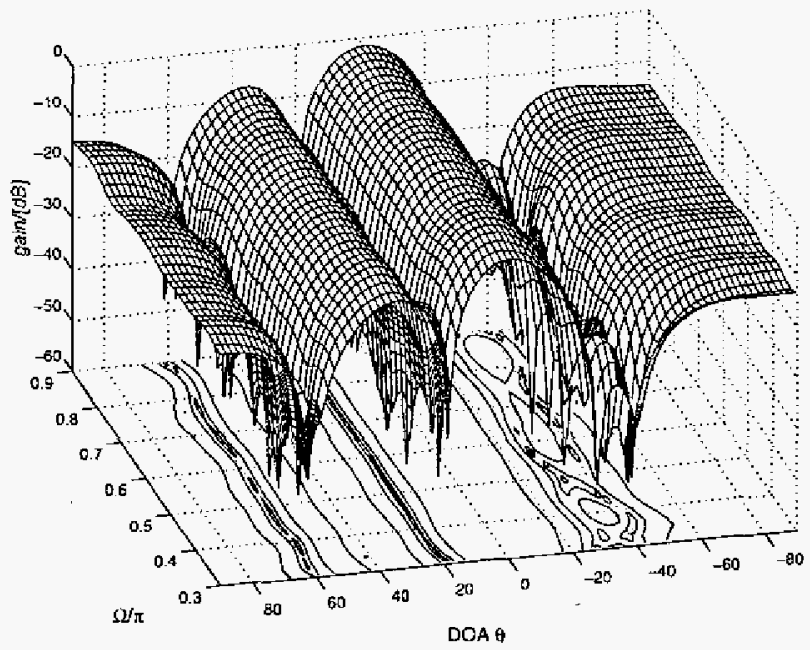

Fig. 6. 3-D beampattern of the proposed GSC over the bandwidth $\Omega \in[0.3 \pi ; 0.9 \pi]$ and DOA range $\theta \dot{\epsilon}$ $\left[-90^{\circ} ; 90^{\circ}\right]$.

$60^{\circ}$ is clearly visible.

\section{CONCLUSIONS}

In this paper, we have proposed a novel structure for broadband adaptive beamforming based on the GSC structure, where the quiescent vector and blocking matrix are replaced by a series of two-dimensional FIFs. These FIFs transform the broadband beamforming problem into a narrowband one and ideally only one adaptive weight is required for each of the FIF outputs. Due to this transformation and the short adaptive filter length, a considerably increased convergence speed is achieved. Additionally, the resultant beam pattern by our proposed GSC also demonstrates a good frequency- invariant property.

\section{REFERENCES}

[1] R. P. Gooch and J. J. Shynk, "Wide-band adaptive array processing using pole-zero digital filters," IEEE Transactions on Antennas and Propagation, vol. AP-34, pp. 355-367, March 1985.

[2] S. Weiss, R. W. Stewart, M. Schabert, I. K. Proudler, and M. W. Hoffman, "An Efficient Scheme for Broadband Adaptive Beamforming," in Asilomar Conference on Signals, Systems, and Computers, Monterey, CA, November 1999, vol. I, pp. 496-500.

[3] W. Liu, S. Weiss, and L. Hanzo, "A subband-selective broadband gsc with cosine-modulated blocking matrix," IEEE Transactions on Anternas and Propagation, to appear in March, 2004.

[4] T. Sekiguchi and Y. Karasawa, "Wideband Beamspace Adaptive Array Utilizing FIR Fan Filters for Multibeam Forming," IEEE Transactions on Signal Processing, vol. 48 , no. 1, pp. 277-284, January 2000.

[5] L. J. Griffiths and C. W. Jim, "An Alternative Approach to Linearly Constrained Adaptive Beamforming," IEEE Transactions on Antemas and Propagation, vol, 30, no. 1, pp. 27-34, January 1982.

[6] K. M. Buckley and L. J. Griffith, "An Adaptive Generalized Sidelobe Canceller with Derivative Constraints," IEEE Transactions on Antennas and Propagation, vol. 34, no. 3, pp. 311-319, March 1986.

[7] O, L. Frost, III, "An Algorithm for Linearly Constrained Adaptive Array Processing," Proceedings of the IEEE, vol. 60, no. 8, pp. 926-935, August 1972.

[8] S. Haykin, Adaptive Filter Theory, Prentice Hall, Englewood Cliffs, 3rd edition, 1996.

[9] Visual Numerics Inc., San Ramon, CA, IMSL Fortran Numerical Libraries, 2002. 